
internationales

vol. 22 - $n^{\circ} 3 \mid 2006$

Turquie 2006 : aux portes de l'Union européenne?

\title{
Immigration familiale turque et activités quotidiennes des femmes : Le souci de réputation dans une cité HLM de la banlieue parisienne
}

\author{
Elif Aksaz
}

\section{(2) OpenEdition}

Journals

Édition électronique

URL : https://journals.openedition.org/remi/3341

DOI : 10.4000/remi.3341

ISSN : $1777-5418$

Éditeur

Université de Poitiers

\section{Édition imprimée}

Date de publication : 31 décembre 2006

Pagination : 155-177

ISBN : 978-2-911627-43-9

ISSN : 0765-0752

Référence électronique

Elif Aksaz, «Immigration familiale turque et activités quotidiennes des femmes : Le souci de réputation dans une cité HLM de la banlieue parisienne ", Revue européenne des migrations internationales [En ligne], vol. 22 - n³ | 2006, mis en ligne le 31 décembre 2009, consulté le 14 avril 2022. URL : http://journals.openedition.org/remi/3341 ; DOI : https://doi.org/10.4000/remi.3341

Ce document a été généré automatiquement le 14 avril 2022

(C) Université de Poitiers 


\title{
Immigration familiale turque et activités quotidiennes des femmes : Le souci de réputation dans une cité HLM de la banlieue parisienne
}

\author{
Elif Aksaz
}

Je remercie Jean-Michel Chapoulie qui a été au cours de cette recherche un guide intellectuel précieux et dont j'ai sollicité l'avis à maintes reprises pendant la rédaction de ce texte qui a bénéficié de sa lecture finale.

1 J'analyserai dans cet article l'univers peu connu des immigrés turcs en France, à partir d'une étude empirique réalisée dans un quartier HLM situé en banlieue parisienne, Les Lilas $^{1}$ où vivent des familles émigrées des zones rurales en Turquie.

2 Je traiterai de deux activités principales que les femmes pratiquent et qui relèvent pour elles de la vie quotidienne : les «activités ménagères » et les "visites de voisinage ». L'importance de ces pratiques est clairement perçue notamment par les hommes, comme le suggère cet extrait d'entretien :

«La vie, ici, est facile pour celles-là [les femmes immigrées turques du quartier]. Celles de là-bas [restées aux villages en Turquie] traient les vaches, elles coupent $d u$ bois $d u$ matin au soir. Ici, il y a tout ce que tu veux, machine à laver, "sèche-linge", "congélateur" [en français dans le discours]. Tout ce qu'elles ont à faire, c'est le "travail de maison" [ev işi : tâches ménagères] et ça, elles en font une "affaire d'Etat"! Bon, il y a ce bébé [en montrant son fils âgé de neuf mois], il faut s'en occuper. C'est tout. "Le reste, c'est $d u$ vide" [gerisi boş: les autres activités sont sans importance, sans utilité]. Celle-ci va chez l'autre, celle-là va chez une autre, les jours passent comme ça, à faire des bla bla [lak lak].» (M., 38 ans, travailleur immigré turc dans le secteur du bâtiment, père de quatre enfants, résidant aux Lilas).

La fréquence des visites que se rendent les femmes turques ainsi que l'importance et le temps qu'elles accordent aux tâches ménagères ont déjà été décrites par Kastoryano (1986) et Timmermans $(2000)^{2}$. Mais orientées par la question de l'intégration, ces analyses se bornent à considérer celles-ci comme une illustration du stéréotype par 
lequel est généralement représentée "la femme immigrée authentique »: femme musulmane au foyer, cloîtrée, respectueuse des normes traditionnelles, doublement soumise dans le contexte migratoire car immigrée par alliance conjugale et victime d'un système patriarcal dont les mécanismes de fonctionnement lui échappent.

Pour rompre avec ces stéréotypes, il ne faut pas chercher à comparer les supposées caractéristiques des femmes turques d'origine rurale, portant le voile, le hijab ou le tchador, à celles qui sont supposées aller de pair avec le statut de femme "émancipée " (Göle, 1993). Il est nécessaire par contre de comprendre le sens que les femmes accordent à ces pratiques, la conception qu'elles s'en font et les circonstances dans lesquelles elles viennent à les concevoir ainsi, en examinant comment elles "définissent leurs situations » (Thomas, 1918).

5 Comme le suggère le témoignage cité ci-dessus, si les femmes turques aux Lilas passent leurs journées les unes chez les autres à « faire du bla bla » et considèrent leurs activités ménagères comme "une affaire d'État " (ou qu'elles les exhibent comme telle aux yeux des hommes), c'est que quelque chose d'important se trouve à l'arrière-plan de ces activités, qui échappe aux autres - ainsi peut-être qu'à leurs maris pour qui ces activités ne font pas sens ou n'ont pas le même sens.

6 Pour saisir le rôle de ces activités dans la vie sociale, je propose d'observer les relations d'interaction des femmes entre elles et d'interroger leurs perceptions de leur environnement social et de leur expérience migratoire.

7 Les visites de voisinage constituent un temps et un espace pour les rencontres journalières entre femmes turques. Chacune raconte sa journée d'activités ménagères, le temps qu'elle y consacre et la manière dont elle procède, fait part des nouveaux produits découverts au supermarché local. Elles se prodiguent des conseils, comparent leur savoir-faire et discourent sur la propreté. Une distinction apparaît en effet très présente dans le discours des femmes : la distinction entre une femme «propre » et une femme «sale ». Une femme "propre» est une femme "bien». Une femme turque me parlant d'une de ses voisines dont nous rencontrons la fille dans le centre commercial me disait par exemple :

"T'as vu celle-là, sa mère, c'est une femme très bien. Va voir chez elle, ça brille, tellement

c'est propre! C'est une femme "titiz" [méticuleuse, soigneuse]. »

Par contraste, «les femmes arabes » (voisines originaires des pays du Maghreb) sont considérées comme "sales». Il ne s'agit évidemment pas ici d'interroger la validité de ces jugements mais de proposer, en s'appuyant sur l'analyse de la construction sociale des catégories des femmes "propres" et des femmes "sales", une description des aspects signalés comme importants de l'univers des femmes turques immigrées d'origine rurale. Je présenterai d'abord le cadre de vie dans lequel s'insère la vie quotidienne des femmes immigrées turques. J'analyserai ensuite la dynamique des pratiques et des discours quotidiens de l'évaluation et de la distinction à travers le critère de la propreté. Enfin j'examinerai le système social auquel ces pratiques et discours appartiennent et à l'intérieur duquel ils trouvent leur sens. 


\section{La structure sociale du quartier et de la population résidente turque}

\section{Un quartier dans et au-dehors de la ville}

9 Le quartier des Lilas est situé à quelques dizaines de kilomètres de Paris. Tout évoque désordre et pauvreté ; papiers et ordures sur les trottoirs, quelques carcasses de voitures, un appartement brûlé il y a un an, des poussettes délaissées en bas des portes, des boîtes aux lettres cassées, des odeurs d'urine dans les ascenseurs... Le quartier des Lilas est une cité HLM qui date des années 1960 : les immeubles aux couleurs délavées sont l'héritage d'une architecture "qui fait songer au milieu carcéral, coupé de tout, coupé $d u$ reste du monde » selon les termes d'un habitant des Lilas. Dans les vingt bâtiments qui composent le quartier, vivent des personnes venues de différents pays, parlant des langues différentes que l'on repère aux vêtements «traditionnels » qu'ils portent. Selon le recensement de 1999, les résidents des Lilas étaient 3392 et en «majorité d'origine étrangère ».

10 Les Lilas est l'un des sept quartiers qui composent la ville de la Vallée ${ }^{3}$. Si la commune est fière de ses six autres quartiers, elle l'est moins des Lilas. Pour les administrateurs de la commune, le quartier des Lilas est «à part ", mais il l'est aussi aux yeux de ses habitants. Ces derniers formulent leur différence par des remarques comme : « Nous des Lilas, les pauvres, les étrangers », «Eux, les gens de la Vallée, les riches, les Français ».

\section{« Nous, les pauvres », « Eux, les riches »}

11 Selon le recensement de 1999, le taux de chômage est de 12,6 \%. Dans la population active de 15 à 24 ans, on comptabilise $27 \%$ de chômeurs; de 25 à 30 ans, $51 \%$; de 40 à 59 ans, $20,8 \%$ et $1,8 \%$ dans celle de 60 ans ou plus. S'agissant de la population active masculine, les taux respectifs sont de $36 \%, 31 \%, 31 \%$ et $1,06 \%$. On remarquera cependant que dans la commune, le taux de chômage était inférieur au taux national $(8,4 \%$ au lieu de $10 \%)$.

12 Le taux de chômage calculé selon les critères de l'INSEE, est cependant à relativiser dans son application à la population du quartier. Un jeune du quartier, d'origine maghrébine, ayant participé au recensement de 1999 en tant qu'agent-recenseur explique :

«Franchement, $y$ a des immeubles qu'on a même pas recensés. On s'en foutait. $Y$ avait un quota pour obtenir un financement, quand on l'a obtenu, on a laissé tomber le reste. Puis, quand c'était fait, c'était un peu "vite fait" quoi, tu vois ce que je veux dire. "

Certaines situations échappent au recensement (comme l'hébergement des membres clandestins de la parentèle ou les populations mobiles, comme les immigrés retraités, effectuant des allers-retours aux pays d'origine). Il faut également tenir compte des fausses déclarations ou des revenus financiers non déclarés. Le taux de chômage aux Lilas peut donc être sur ou sous-évalué. D'après les habitants, qui désignent le quartier comme "le quartier des pauvres, des prolétaires", le niveau de vie y serait bas à la différence des autres quartiers de la Vallée qu'ils décrivent comme " la ville des riches, la ville bourgeoise ». Cette distinction prend une autre forme qui n'est pas uniquement fondée sur des critères socio-économiques mais sur celui de l'appartenance nationale. 


\section{« Nous, les étrangers », " Eux, les Français »}

à penser que le quartier des Lilas est exclusivement peuplé d'étrangers. La population étrangère dans la commune est répartie comme suit: 26746 habitants dont 2509 étrangers ; 956 personnes étrangères résideraient aux Lilas soit 38,1\%. Mais rapportés à l'ensemble de la population des Lilas, les étrangers ne représentent que $28 \%$. En effet, cette proportion de résidents étrangers aux Lilas, excluant par définition les personnes naturalisées françaises, sous-évalue la part de ceux qui, aux yeux des habitants, restent des étrangers. Naturalisé, l'immigré ou son enfant n'est pas un «Français » mais un « étranger » qui « a ses papiers ». Quand les habitants turcs et maghrébins évoquent un «Français » ou un « Gâvur »", ils désignent un habitant de la ville ou d'ailleurs mais pas quelqu'un des Lilas qui est un « un quartier d'étrangers ».

Les habitants reconnaissent la présence dans le quartier de personnes qui ne sont pas des « étrangers ». Celles-ci situées à la frontière - «Français » habitant les Lilas - ne seraient ni des « étrangers », ni des « Français »; mais des « cas sociaux » :

"C'est pas des vrais Français. C'est des "cas sociaux". Les vrais Français, ils vivent pas dans la cité. Nous, on est là parce qu'on est étranger. Nous, on nous a mis là, dans ces cages à poule, parce qu'on n'est pas chez nous. Si les Français les ont mis avec nous, c'est qu'ils sont vraiment pires que nous! (Un jeune homme d'origine turque).

Désigner et dénigrer par l'appellation de «cas sociaux» «les autres parmi nous» permet aux habitants la gestion d'une image stigmatisée qu'ils pensent avoir aux yeux des « autres » (« les Français ») :

«Pour eux [les gens de la ville], les Lilas, c'est la tache de la Vallée. Pour eux, c'est l'Afrique ici. Quand t'es des Lilas, ça y est, t'es qu'un "immigré”, t'es qu'une merde à leurs yeux. " (Une femme d'origine algérienne).

"Les gens de la ville, ils pensent quoi de nous? Ils nous montrent du doigt. Ils disent que c'est de là que vient la merde, la délinquance. Le Q.G. [quartier général] de la délinquance. C'est vrai qu'il y a des délinquants mais on est pas tous des délinquants. C'est vrai qu'on est pas des " $p$ 'tits bourges" de la ville. Ils veulent l'intégration du quartier, des immigrés, mais est-ce qu'ils veulent de nous? Ils nous voient comme des fouteurs de merde. S'ils nous voient comme ça, nous aussi, on veut pas d'eux ». (Un jeune homme d'origine marocaine).

Ainsi la perception de la vie du quartier comme étant exclusivement réservée aux immigrés est maintenue. Dès lors, la présence du « Français » dans le quartier revient à lui ôter son caractère « français » car on ne peut pas être à la fois pauvre et « français ».

\section{Les résidents turcs}

Le recensement ne permettant pas de disposer de données fiables selon les origines géographiques, j'ai pu constituer un micro-recensement des Turcs du quartier ${ }^{5}$. Parmi les « étrangers » vivant dans le quartier, il existe en effet un ensemble d'individus que d'autres appellent « les Turcs » et qui se définissent eux-mêmes comme «les Turcs ${ }^{6}$. Ce groupe comprend 156 personnes formant 31 ménages. Il n'existe pas de ménage turc composé de célibataire(s) : tout ménage turc aux Lilas est composé de famille nucléaire et élargie : 29 ménages sont formés de parents et d'enfants célibataires ; un ménage comprend des parents vivant également avec leur fils âgé de 23 ans et son épouse de 18 ans ; un ménage comprend une jeune fille de 15 ans, mariée, habitant chez ses parents, dont le conjoint turc n'est pas encore arrivé de Turquie. Donc, en tenant compte du cas particulier de cette jeune fille, on peut conclure que la population turque aux Lilas est 
composée de 65 individus mariés (64 conjoints formant les 32 couples et la jeune fille mariée). Le nombre de célibataires s'élève à 91 et représente $58,3 \%$ de l'ensemble de la population turque. Ces célibataires sont âgés de moins de 27 ans : 85 ont moins de 20 ans. ${ }^{7}$ Parmi ces 91 célibataires, figurent 46 garçons et jeunes hommes et 45 fillettes et jeunes filles; parmi les jeunes filles, il y en a 4 (entre 15 et 19 ans) qui sont fiancées et une (16 ans) qui est sözlü (" promise ») $)^{8}$ à un jeune homme se trouvant en Turquie.

La majorité des hommes mariés travaillent dans le secteur du bâtiment (ouvrier, artisan, maçon, plombier). D'autres exercent les professions d'éboueurs, livreurs, ouvriers dans une boulangerie industrielle, employés d'une usine pharmaceutique ou à Auchan. Les hommes qui n'ont pas été scolarisés en France sont majoritairement sans diplôme ou diplômés de l'école primaire en Turquie. Le plus diplômé a obtenu un diplôme de niveau secondaire en Turquie (après des études au lycée, dans la région de son village d'origine). Quelques hommes scolarisés en France ont suivi des études dans un lycée professionnel sans obtenir de diplôme. Les jeunes les plus scolarisés sont au lycée professionnel (CAP, BEP) alternant parfois travail et études. Certains ont arrêté les études à ce stade ou avant. La majorité des jeunes travaille dans le même milieu professionnel que leurs pères et quelques-uns dans les commerces à proximité du quartier (dans le service de sécurité à Auchan et dans une discothèque).

Les femmes mariées ont un niveau de scolarisation similaire à celui des hommes mariés. Hormis les deux femmes ayant fréquenté le lycée en Turquie dont l'une a obtenu le diplôme de fin d'études, les autres ont au plus fréquenté l'école primaire en Turquie. Les quelques femmes qui ont été scolarisées en France possèdent un CAP de comptabilité. La grande majorité n'exerce pas d'activité rémunérée. Certaines ont cependant travaillé à leur arrivée en France, sans être déclarées, dans «l'atelier de confection familial » dans le sous-sol du pavillon des beaux-parents. Celles qui exercent actuellement une activité professionnelle rémunérée le sont dans les commerces à proximité (caissière à Auchan, employée chez Flunch). Parmi les jeunes filles célibataires, les plus instruites sont les deux sœurs d'une famille, qui préparent un CAP et travaillent à temps partiel. Les autres sont scolarisées à des niveaux inférieurs ou ont arrêté leurs études avant d'atteindre ce niveau de scolarisation.

\section{Les visites de voisinage et les tâches ménagères}

21 Lors de mes premières rencontres avec les femmes turques du quartier, la conversation débutait ainsi :

"Bonjour, comment allez-vous, que faites-vous?"

"Que faire? Toujours pareil! "La propreté", le repassage, la lessive, les enfants... ça ne s'arrête jamais. Nous passons notre temps à faire "le travail de maison" ".

"Mais vous ne vous arrêtez jamais?»

«Si. Si. On s'arrête [rires]. "Ici chez nous" [dans notre quartier], quand "le travail de maison" est fini, nous, les femmes turques, nous allons "les unes aux autres pour le misafirlik"[ " pour se rendre visite »]. On boit du thé, on discute. Ce n'est que là qu'on se repose."

Aux Lilas, la vie quotidienne des hommes turcs se déroule principalement en dehors du quartier. Ils vont sur les chantiers à Paris, en banlieue proche ou dans d'autres villes. Les femmes me disent alors: "Le mien est en "déplacement" [en français dans le discours], viens [chez-moi] quand tu veux». Que leurs maris soient "en déplacement » ou non, sur les lieux de travail ou de divertissement (au café pour certains ${ }^{10}$, en 
discothèque pour d'autres), ceci n'a pas de répercussion sur la nature des activités quotidiennes que poursuivent leurs épouses. Deux choses préoccupent celles-ci : «faire le ménage » et "aller les unes chez les autres ». Il est rarissime pour une femme turque aux Lilas, qu'une journée se déroule sans qu'elle n'ait effectué des activités ménagères et visité ou reçu chez elle une voisine turque notamment quand son état de santé ne lui permet de se déplacer. Ses voisines viendront lui souhaiter «geçmiş olsun» («bon rétablissement »).

Aux Lilas, les pratiques ménagères sont présentées à la fois comme une obligation " faire le travail de maison » et comme une activité consistant à « faire de la propreté ». Les visites de voisinage sont perçues comme un temps hors travail, librement vécu. Les femmes se réunissent chez l'une ou l'autre et conversent tout en buvant du thé turc et en mangeant des gâteaux ${ }^{11}$. Ces deux activités - le ménage et les visites - sont très fortement reliées. Faire le ménage est conçu comme un véritable travail alors que les misafirlik sont des moments de rencontre organisés où se constituent et s'entretiennent des réseaux d'interconnaissance.

\section{« Ici, tout le monde se connaît »}

Comme diverses études sur les cités HLM l'ont souligné (par exemple Lepoutre, 1997), le quartier des Lilas est un milieu d'interconnaissance. On me disait en effet souvent: "Tout le monde se connaît ici». La plupart des femmes maghrébines affirment: «Les femmes turques, on ne les connait pas ici. Ce sont comme des Chinois pour nous. Pourtant nous sommes toutes des musulmanes». Celles qui affirment connaître des Turques ignorent souvent les prénoms de celles-ci, les désignant simplement comme "des Turques" ("Dans mon bâtiment il y a une Turque »). Aux débuts de ma présence dans le quartier, alors que la communauté turque n'était pas informée de ma présence et du fait que j'étais turque, j'ai observé les comportements distants des femmes turques à l'égard de toute personne non turque. Mme F., une Maghrébine, commente ainsi :

« Tu as vu comment elles [les femmes turques] sont? Ni bonjour, ni bonsoir. Elles sont toujours entre elles. Elles méprisent les autres. Même quand les enfants se chamaillent, elles leur courent tout de suite après, pour les récupérer. »

$\mathrm{M}^{\mathrm{me}} \mathrm{T}$., qui estime être la seule Maghrébine à entretenir des relations avec les Turques en raison de l'amitié de son fils avec B., un jeune turc du même âge, ne connaît pas les prénoms de celles qu'elle salue dans le quartier ou dont elle me parle. Elle est une des locataires les plus anciennes des Lilas (depuis 24 ans) et fut voisine de la mère de B. durant les seize années où leurs fils furent amis de palier et amis de classe. Ce qui n'empêche pas $\mathrm{M}^{\text {me }} \mathrm{T}$. de décrire leur relation ainsi :

«La mère de B. est une femme très froide. Elle a l'air dur. Elle a commencé à dire petit à petit: "Bonjour". Mais on ne s'est jamais fréquenté. Mais les Turques, elles sont toutes comme ça. Elles sont entre elles. »

Lorsque les femmes turques parlent de leurs voisines maghrébines, elles utilisent le qualificatif « Arabes » :

$\mathrm{R}:$ «Je ne sais pas qui c'est, c'est une Arabe. »

E. A : «On dirait une Algérienne. »

$\mathrm{R}$ : «Qu'est-ce quej'en sais, c'est une Arabe, quoi!»

En effet, tout le monde ne se connaît pas de manière individualisée et il ne suffit donc pas d'habiter le quartier pour se connaître. À l'inverse de cette quasi-absence d'information et d'intérêt au sujet des Maghrébines, les femmes turques se connaissent 
et savent jusqu'au nom des villes et parfois des villages d'origine respectifs. Le degré d'interconnaissance observé parmi ces femmes est en effet intense. Elles détiennent les unes sur les autres des informations, à la fois générales et précises tout aussi formelles qu'informelles : la date d'arrivée dans le quartier, les noms, prénoms, l'âge des enfants ( Il aura cinq ans dans quatre mois »), le temps restant pour l'accouchement... enfin toute une série d'informations sur les évènements survenus dans la vie des autres turques du quartier. Ces informations leur servent par ailleurs de référence pour se repérer dans le temps : aussi dans une conversation, une femme dit: "Quand c'était ?... Les C. (la femme C. et sa famille) venaient d'acheter leur voiture (cet événement vécu par la femme voisine C. n'entretient aucune relation avec le sujet qui fut abordé par la suite en dehors du fait d'être survenu au même moment), c'était il y a quatre ans, nous... ». Il arrivait que les informations données sur un fait par plusieurs personnes diffèrent l'une de l'autre, mais les écarts étaient rarement importants.

Dans le quartier, l'information circule rapidement, se propage et atteint toutes les Turques à des degrés différents. Dans ce milieu de forte interconnaissance, chacun est plus ou moins conscient du rôle d'informateur qu'il joue vis-à-vis des autres. Les visites de voisinage rendues entre femmes turques permettent la circulation d'informations et un certain contrôle des femmes sur leur entourage.

\section{L'organisation informelle des visites}

Les visites de voisinage correspondent à un mode de sociabilité qui inclut des liens d'amitié et de solidarité, l'échange de biens et de service, alternatifs aux relations de parenté dans le quartier ${ }^{12}$. Ces visites respectent un code social précis et bien connu des participantes :

« Hatice "abla"13, il faut aller chez les nouveaux venus, n'oublie pas. »

« Oui, oui, je l'ai en tête [j'y pense], maisj'attends que mes invités soient partis [son cousin

et la famille de celui-ci sont venus de province et sont restés trois jours à la maison]. »

Ainsi cette famille, les «nouveaux venus », a aménagé dans le quartier vers la fin de ma présence et la visite de «bienvenue » évoquée ne s'est pas effectuée avant mon départ. Cependant, les femmes m'ont racontée qu'elles avaient reçu, les jours suivant leur emménagement, des visites et des cadeaux de la part des femmes turques du quartier; ce qu'elles interprètent comme l'expression de "l'accueil chaleureux" et de "la solidarité » à leur égard. Lors de la première visite appelée « aller/venir pour (souhaiter) la bienvenue ", il s'agit de proposer à la nouvelle arrivée de faire partie de la communauté. Ainsi « la nouvelle » a désormais le droit d'aller chez celles qui lui ont rendu visite, droit qui lui est rappelé par la formule de politesse : «viens toi aussi chez moi, c'est désormais à ton tour ». Il s'agit aussi d'une première mise à l'épreuve ${ }^{14}$; cette première visite offre à la nouvelle venue la possibilité de manifester sa volonté de s'intégrer au groupe. Si elle veut prendre place dans le système des relations sociales, deux devoirs lui incombent: répondre aux invitations et exprimer le souhait de recevoir. Aux Lilas, personne n'a jamais refusé la réception de la visite de bienvenue, comportement qui serait perçu comme un refus du système de relation.

Par le biais du misafirlik, institution par laquelle se crée le lien ou le droit de passage d'un foyer à l'autre, les nouveaux arrivants sont intégrés dans la communauté turque locale et le cercle d'interconnaissance est élargi. Une fois intégrée dans le système du 
misafirlik, la «nouvelle » choisira les femmes du groupe avec qui elle entretiendra des relations privilégiées. La visite rendue/reçue est pour les femmes une occasion de s'évaluer mutuellement :

"Que j'y aille, que je regarde [chez les voisins nouveaux venus]. Qui sont ceux-là? Ils sont "quoi de qui"? [kimin ne'siymiş ? : la formule interrogative employée pour situer un individu dans un système de liens de parenté (la fille d'un tel, beau-frère d'un tel), est utilisée ici dans un sens plus large et plus courant, se rapportant à tout type d'information sur la personne)]. »

En dehors des visites formelles rendues pour souhaiter la bienvenue dans le quartier et de celles qui sont rendues de manière périodique ou occasionnelle [comme suite à une maladie ou le retour en France après un voyage au pays d'origine], les visites quotidiennes se pratiquent selon les affinités. La manière dont celles-ci se créent et se tissent entre femmes à travers les relations de misafirlik est complexe. Une règle essentielle est que les visites doivent être rendues à tour de rôle :

«Ici, c'est bizarre, "vallah" [le jurement en turc dans le discours en français]. Si ma mère va chez quelqu'un avec une copine à elle, après, c'est à elle [la personne chez qui elles sont allées] de venir chez nous et chez sa copine. C'est "chelou" ["louche" prononcé en verlan qui signifie "bizarre"] ici. On va pas deux fois [de suite] chez la même personne. » (P. 17 ans, jeune fille d'origine turque).

Le principe de la réciprocité instaure un système de droits et d'obligations mutuelles qui garantit un échange équitable : recevoir une visite offre le droit d'en rendre une ${ }^{15}$. Mais on peut décider de ne pas user de ce droit (et donc de ne pas respecter l'obligation) et de transformer l'autre en un obligé (qui a reçu l'hospitalité mais qui ne peut la rendre et qui reste redevable):

Hayriye [avec une certaine fierté] : «La "Sivaslı" [une femme habitant le quartier, émigrée du département de Sivas] m'a "envoyée une information" [un discours que la "Sivaslı" a tenu en son absence mais pour que cela lui soit transmis]... Tu te souviens, celle qui était chez Nuriye, l'autre fois... Me disant ["me reprochant"]: "Pourquoi Hayriye ne vient pas chez moi ?". »

Si ne pas recevoir la visite qu'elle attend peut être humiliant ${ }^{16}$ pour une femme (dont on refuse l'hospitalité et que l'on traite comme quelqu'un qui n'est pas une égale), cela constitue néanmoins un défi qu'elle peut relever dans le temps, puisque la règle de la réciprocité est atemporelle. En attendant la visite qui la fera reconnaitre comme une égale, elle ne peut pas se rendre chez l'autre - ce qui signifierait admettre son infériorité. Elle ne peut pas non plus réclamer la visite - demander à quelqu'un d'accepter son hospitalité alors qu'il le refuse est rabaissant. Certaines peuvent alors recourir à la médiation du groupe. Pour rapprocher deux femmes, A et B, dont les relations se sont refroidies, les membres du groupe peuvent intervenir et les inciter à se rendre visite. Mais $\mathrm{A}$ peut considérer que répondre à une visite demandée par les autres, que B ne lui a pas exprimée personnellement, n'est pas digne d'elle et peut discréditer sa réputation au sein de groupe :

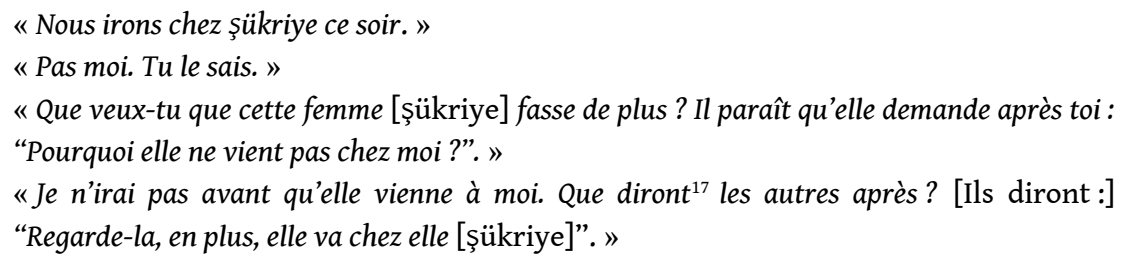

Ce qui rend effective la relation de misafirlik est aussi bien l'estime qu'une femme pense qu'une autre lui porte (la manière dont elle est reçue en est une base d'évaluation) que 
l'estime qu'elle a de l'autre. Celles qui se fréquentent sont vues par le groupe comme des membres suffisamment proches pour être à la fois compatibles et compétitifs ${ }^{18}$. Les visites quotidiennes entre femmes se pratiquent dans un contexte où la réputation de l'une au sein du groupe engage celle de l'autre ${ }^{19}$. Savoir à qui rendre visite, qui recevoir, sur quel mode, à quelle fréquence, qui éviter de recevoir etc. sont des questions particulièrement importantes pour les femmes et leur réputation en dépend.

Mon entrée chez les femmes turques est une illustration de ce "jeu social de la réputation ». Il m'a fallu un long travail pour pénétrer l'univers des familles immigrées turques dans lequel j'étais considérée comme " une étrangère pas "gâvur" " ". J’ai entendu les habitants turcs employer à mon sujet d'autres qualificatifs comme : "Une fille loin de sa famille», "La fille de notre maison», "La fille du quartier ", «Étudiante», Écrivain", "Jeune fille à marier ", "Une "sosyetik" " ${ }^{21}$ autant de dénominations qui étaient selon les situations des avantages ou des inconvénients pour la réalisation de ma recherche. ${ }^{22} \mathrm{~J}$ 'ai passé des mois dans le quartier des Lilas, où j'ai été hébergée par une famille algérienne, à chercher à nouer des contacts avec les habitants turcs; les jeunes hommes turcs du quartier étant les plus « disponibles» pour discuter avec moi. Puis, progressivement les familles turques m'ont ouvert la porte de leur foyer une par une. J'allais comprendre par la suite que cette "acceptation » était pour elles une manière de gérer la présence compromettante dans leur quartier d'une "jeune fille turque... inconnue... qu'il se trouve qu'elle est venue dans le quartier... hébergée chez des "Arabes"... parlant dehors avec les garçons». Il fallait pour les familles turques savoir quoi faire de cette étrangère pour « se garder du danger » qu'elle représentait à leurs yeux : «[...] ou bien refuser de l'admettre, la chasser et l'éloigner [...] ou bien, si l'on consent à la recevoir, la socialiser, c'est-à-dire la séculariser » en transformant "l'étranger hostile en hôte " (PittRivers, 1997 : 160). J'ai eu l'occasion de recevoir, bon nombre de fois, des invitations de la part des femmes, parfois indirectement par le biais de leurs enfants jouant dehors ou leurs époux que je rencontrais dans le quartier: "Ma mère t'attend", "Ma femme t'appelle ». Inquiète au début par l'urgence de ces appels, je leur demandais naïvement pourquoi j'étais «appelée " par leur mère ou leur épouse. Les hommes et les enfants répondaient tout aussi naïvement : "Je sais pas, elle m'a dit : "Si tu la vois, dis-lui de venir à la maison" ". Certaines femmes me faisaient également des reproches: "Viens dormir chez nous, depuis deux nuits, tu restes là-bas!». Mais ces invitations survinrent surtout après ma réception préalable chez des «meneuses " puis mon hébergement dans une famille. Une d'entre elles, prenant ses précautions, avait bien ressenti le besoin d'exprimer par la parole les "devoirs» ou les responsabilités que m'assignait le «droit» de rentrer chez elle :

"Désormais, tu "rentres chez nous et tu sors de chez nous", tu es devenue en quelque sorte la "fille de notre maison" [un membre de la famille]. Tu peux rester tant que tu veux, tu mangeras de ce que nous mangeons. À partir de maintenant, ton namus ${ }^{23}$ est aussi le nôtre. Ils [les autres habitants turcs du quartier] diront: "Cette fille rentre chez eux et sort de chez eux". »

37 Si le misafirlik assure le fonctionnement d'un réseau féminin de production et d'échange d'informations, de services, de biens, et de contrôle social, il constitue aussi un terrain où se négocient à l'intérieur du groupe les réputations et les statuts des membres. 


\section{Ne pas recevoir les « Arabes »} les activités ménagères. Dès mon entrée dans leur domicile, celles-ci s'excusaient du désordre. Le salon me semblait pourtant plus que présentable. Devant mon étonnement à l'écoute de ces excuses, l'époux d'une des femmes, ouvrier dans le bâtiment, avait complimenté ironiquement son épouse ainsi : "Nous, on a fini [la construction d'] une maison, notre "Madame" [en français], elle n'a pas fini [le ménage de] la nôtre! [Rires mutuels]. » Le fait de participer aux tâches ménagères m'avait permis de voir l'arrièreplan social de "la propreté». Ainsi Raziye ordonnait à sa fille (13 ans): "Fais vite, regarde-moi ça, combien de fois, je t'ai dit de faire ça comme ça? Cette fille ne comprend pas si je ne la frappe pas!». La jeune fille restait silencieuse et soumise en poursuivant son 
travail, mais les menaces de sa mère ne cessaient pas: "Dépêche-toi, sinon je vais te frapper!» suivi d'un clin d'œil et d'un sourire discret en se tournant vers moi. Au bout d'un moment les pressions maternelles cessèrent et la jeune fille se rendit dans sa chambre alors que sa mère et moi débarrassions la table et vidions la machine à laver la vaisselle. La jeune fille, Seçil me dit plus tard :

Seçil : "Pfff! Comment elle s'la pète devant toi! Elle fait trop "tièpe"! ["Pitié" prononcé en "verlan" insinue que les comportements de sa mère lui paraissent ridicules]. "Seçil, fais ça! Seçil, ramasse ça!". Genre! Quand y a personne, elle s'en fout. »E. A : « Tu ne fais pas le ménage quand il n'y a personne? "Seçil : "Si. Je fais le ménage et ça me fait chier [rires] mais devant les gens, elle [sa mère] me fait passer pour une bonne. »

« Être propre » signifie ainsi " paraître propre aux yeux des autres »; et la "propreté » est une valeur que l'on voudrait que les autres pensent qu'une femme a bien transmise à sa fille. Les misafirlik constituent sans doute l'espace et le temps privilégiés de la mise en scène de la propreté. Mais penser que deux femmes turques se rendent visite parce qu'elles se considèrent mutuellement "propre ", serait aussi erroné que de considérer que les Turques ne fréquentent pas les Maghrébines parce qu'elles les trouvent " sales ». La "propreté » permet de justifier publiquement une fréquentation lorsque les parties considèrent qu'il est nécessaire de le faire et de justifier entre pairs et de s'encourager à la non-fréquentation des Maghrébines.

\section{La propreté : un critère de distinction}

\section{Devenir « polie »}

Les familles immigrées turques vivant au quartier des Lilas sont originaires des köy (le mot «village » est prononcé en turc dans les phrases formulées en français) ${ }^{26}$. Seules deux familles viennent d'Ankara, mais elles ne sont pas considérées par les autres comme étant des şehirli (citadin) du fait qu'elles ont immigré de leur village vers cette ville. Les avantages sociaux (sécurité sociale, allocations familiales etc.) le fait de travailler et de mener son existence en France sont valorisés : "On est venu ici, on est devenu quelqu'un. Regarde, on a des médecins qui nous soignent, des écoles pour les enfants. Même pour les enfants, ils donnent de l'argent ». Même pour celui qui travaille en dehors de toute réglementation dans le secteur informel, l'expérience migratoire est tout de même prometteuse : "On est venu gagner notre pain. Sinon, pourquoi on serait là ?». Les femmes attribuent à leur expérience migratoire une signification particulière qui va au-delà des aspirations matérielles comme l'illustre le récit de Sevda, une femme au foyer âgée de 37 ans et mère de quatre enfants. Elle est arrivée en France, il y a dix-sept ans, après avoir épousé un homme vivant en France. Elle a fréquenté l'école primaire en Turquie, dans son village natal. Les parents de son époux sont originaires d'un autre village situé à proximité. En arrivant en France, Sevda s'est installée, comme les autres brus venues de Turquie, chez les parents de son mari, «le temps de faire des économies». À l'issue de quatre années de cohabitation conflictuelle avec sa belle-mère, Sevda a déménagé dans le quartier des Lilas.

"Comment j'ai connu mon mari? [Elle déduit de cette question mal formulée que je souhaite connaître comment elle s'est mariée avec lui] Tu sais "chez nous là-bas" ["bizim oralarda" signifie le lieu d'origine et se différencie à la fois de "bizim burada" ("ici, chez nous") qui signifie "dans le quartier" et de "bizde" ("chez nous") employé pour désigner "chez nous, les Turcs en général"], ces choses se font, comme on dit, à la "görücü" [traduit en français comme "mariage arrangé"]. Il paraît que la mère de celui 
[la mère de son mari] m'a vue [l'usage de "il paraît que" permet d'exprimer l'extériorité de Sevda au déroulement des événements], que je lui ai plu. Il paraît qu'ils sont venus me demander [en mariage]. Ceux-ci [la famille de son époux] étaient $d u$ village proche. Moi, je suis "venue à eux" comme bru ["ben bunlara gelin geldim": intégré la belle famille comme un nouveau membre de la famille et du foyer]. Tout a commencé comme ça... Moi, je les croyais des gens "kibar" [polis, raffinés, courtois, distingués]. Je me disais: "Ils ont vécu tant d'années en France, ils doivent être 'autres' [différents], ils doivent 's'asseoir et se lever autrement' ["oturmaları kalkmaları bi başkadır" : comportements valorisants qui selon elle, les distingueraient de ceux qui n'ont pas émigré du village]." Je me disais: "Il faut que je fasse attention à comment je m'assoie et me lève avec eux" [en leur présence]. Hélas! Je suis arrivée, j'ai vu combien ils étaient "kaba" [rustres, grossiers]. Ils étaient tous si mauvais, si ignorants. Ils pensaient qu'ils savaient les choses. Moi, je me taisais. Je ne disais rien. Je disais: "Oui, mère" [à sa belle-mère], "oui, Zuhal” [à sa belle-sœur]. Je savais que ce qu'ils disaient était faux, le juste était ce que je disais, moi. Mais je ne leur disais rien. Un exemple? Un jour, j'ai cuisiné des pâtes à la sauce tomate. L'un a dit: "Je n'en mangerai pas", l'autre a dit: "Je n'en mangerai pas". Personne n'a mangé. Ils ne connaissaient pas. Ils ne connaissaient rien. Nous, dans ma famille, on a toujours mangé comme ça, on a vu comme ça. Ici [en France], ceux-ci n'ont "rien vu" [connu]. Nous, on était au village, mais ma mère nous habillait bien, "nos habits et notre tête étaient propres" ["üstümüz başımı temizdi”]... Quand je l'ai vu la première fois [son époux], j'ai dit à ma mère: "Mère, celui-ci a le visage d'un gamin. C'est lui qui va 'm'approprier'?" ["sahip çıkmak" : être capable de prendre soin d'elle, de répondre à ses besoins et avoir le courage de la protéger contre la malveillance des autres]. Soit, il était en France. Mais l'autre [un autre candidat en mariage] était mieux. Il n'était pas en France, lui. Il était au bourg, mais il était "gururlu" [un homme possédant de la fierté]. Enfin, il m'avait l'air de quelqu'un "gururlu". Mon père avait dit à tout le monde: "Je donnerai ma fille à la ville, je ne la donnerai ni au bourg, ni au village" ".

Le qualificatif «poli » qui s'oppose à « rustre », « ignorant » et à görmemiş (« celui qui n'a pas vu ", qui n'a pas connu de nouvelles choses, qui n'est pas sorti de son milieu ou de sa condition sociale) est également employé pour décrire les « Français » et les şehirli (" citadins» turcs). L'immigration représente une expérience d'ascension sociale qui suppose que l'immigrant va sortir de sa condition sociale et acquérir les caractéristiques attribuées aux «Français » et aux « citadins ». Pour prétendre à cette mobilité, les candidats au mariage (futures brus et futurs gendres) vivant au village doivent aux yeux de leurs éventuels futurs beaux-parents et de tous ceux qui pourraient influer le jugement de ces derniers, posséder certaines qualités qui les distinguent des autres candidats du village. Une expérience de mobilité spatiale et sociale antérieurement vécue peut constituer un élément de distinction et de sélection. Il en va sans doute ainsi pour le plus diplômé des hommes aux Lilas ayant suivi un cursus secondaire arrivé de Turquie pour épouser une immigrée. Néanmoins pour les futures brus, un autre critère de sélection consiste à avoir la réputation d'être "propre ». La valeur attachée à la "propreté " est très importante et doit être rapportée aux autres critères de distinction qui attribuent à l'individu et à sa famille une certaine position dans la hiérarchie sociale locale. Sevda raconte :

"Ma mère n'avait pas "lu" ["okumamış" : illettrée] mais elle avait lu "le livre de la vie" [la vie, telle qu'elle a menée, a fait d'elle une femme ayant des connaissances]. Elle était respectée au village. Tous les villageois venaient lui "demander l'esprit" [ses conseils, son point de vue]."

"Les enfants de notre village ne "lisent" pas [ne vont pas à l'école primaire ou ne poursuivent pas leurs études au-delà]. Mon père avait envoyé mon frère aîné à Bursa [une grande ville en Turquie] pour qu'il "lise". Maintenant, il est comptable à Bursa. Il a "pris à côté de lui" [il a fait venir à Bursa] mon frère cadet. Ils ont ouvert ensemble un 
bureau de comptable. Ils ont "pris à côté d'eux" mes parents. Maintenant, ils sont tous à

Bursa. » si le ou la futur(e) conjoint(e) réside au village. Si le fait d'être originaire d'un village donné peut se révéler un handicap ou un atout pour la réputation d'une femme (selon les préjugés que les autres ont à propos de son village, de la région, du département ou du district), ses köylü (femmes originaires du même village) peuvent aussi influer sur sa réputation. Il reste cependant que celle-ci n'est pas uniquement liée aux « dires » des autres et dépend aussi du contexte ${ }^{29}$. Les femmes qui sont au village considèrent, de la même manière que celles des Lilas, les «dires » des autres quand elles évaluent leurs éventuelles futures dünür (positions réciproques dans le système des relations de parenté de deux femmes dont les enfants sont unis par une alliance conjugale). En veillant à sa réputation au sein du quartier, une femme peut penser veiller à celle qu'elle a aux yeux des gens de son village puisque l'information peut y être transportée par ses köylü résidant aux Lilas (ou résidant ailleurs), qu'elle ne connaît pas forcément elle-même, mais qu'une voisine connaît à travers par exemple un membre de sa parentèle qui habite dans le même quartier que celles-ci ${ }^{30}$. Dans un contexte où des liens permanents et forts sont entretenus avec les villages d'origine (aide financière, investissements immobiliers, allers-retours périodiques au village et arrivée des gens du village en qualité de main-d'œuvre ou de gendre et bru), les réputations des femmes et de leurs filles vont au-delà de leur quartier de résidence ${ }^{31}$. 


\section{Le recrutement de la main-d'œuvre à l'intérieur de la communauté}

51 La " propreté » constitue également un critère pris en compte lors d'un recrutement de la fille, du fils et de l'époux d'une femme. Les relations professionnelles associant des habitants turcs ressemblent à une toile d'araignée. Ainsi, dans les dossiers de candidature pour un poste de caissière à Auchan, figure la question suivante: "Connaissez-vous une personne qui a déjà travaillé à Auchan? ". Il n'est pas demandé de qualifications pour les types de postes occupés par les habitants turcs et les grandes structures commerciales implantées dans les périphéries sont conscientes du contrôle social qu'exerce le réseau communautaire sur l'individu. Le recrutement qui s'appuie sur des relations constitue une garantie et permet un mode de gestion de la maind'œuvre sans qualification comme le montre l'exemple ci-dessous :

Süleyman qui occupe un emploi dans le bâtiment a travaillé auparavant d'abord à Auchan pendant dix ans et par la suite chez Flunch. Il a fait entrer deux de ses sœurs, la troisième est employée chez Flunch depuis vingt-deux ans. Celle-ci a permis l'embauche de deux de ses filles et d'une voisine turque du quartier :

"C'est des filles bien, c'est des filles de bonne famille. Quand tu vas chez elles, elles ne laissent rien à faire à leur mère, elles travaillent comme des fourmis. »

53 Nermin travaille chez Flunch depuis deux ans. Elle a permis à Sevim, qui habite le quartier, d'être recrutée. Après avoir rempli ensemble le dossier d'embauche et inscrits les deux noms, Nermin dit de Sevim :

"Sevim est une fille bien. C'est une fille raisonnable. On n'a jamais entendu des choses sur elle. C'est une fille "hanım" [bien élevée] et tu as vu, elle est très "becerikli" ["habile" suggérant la participation de Sevim aux activités cuisinières (service, vaisselle, etc.)]. "

Il existe aussi des cas où l'avis donné n'est pas favorable :

"Après mon entretien d'embauche, on a demandé à quelqu'un à Auchan à mon sujet, "ismi bende kalsın" [“je garde son nom pour moi”], il a donné un avis défavorable. » (Zeliha, 31 ans, mère de deux enfants).

On peut penser que cet avis donné pour le recrutement de Zeliha est lié à des relations conflictuelles avec sa famille. Alors qu'une procédure de divorce avec son mari était entamée, Zeliha s'est réconciliée avec lui. Il a rejoint le foyer, mais cette situation a été vécue par ses parents comme un ayıp ${ }^{32}$ : "Tu restes séparée d'un homme pendant un an et demi et maintenant tu "retournes dans son lit" (koynuna girmek), c'est ayıp vis-à-vis des autres (elegüne karşı)!». Pour Zeliha, cette réconciliation ne convenait pas à ses parents parce que durant la période de séparation, elle avait pris en charge les besoins quotidiens de ses trois frères cadets en plus de ceux de ses deux enfants : "Bien sûr, ça les arrangeait mes parents. Mais moi, j'ai dit: "ça suffit", je préfere m'occuper d'un seul [le père de ses enfants], que de trois [ses frères] ». Le fait que deux de ses frères travaillent à Auchan a pu jouer un rôle dans l'échec de sa demande d'embauche.

L'évaluation entre femmes et la réputation qui en découle pour chacune influence aussi parfois le recrutement de leurs maris. Le couple Kadriye et Kazım accueille un autre couple, Dursune et Hüseyin, pour le thé. Alors que les deux femmes Dursune et Kadriye sont habituées à se rendre visite, c'est pour la première fois que Hüseyin se rend chez Kazım ; la veille, Kadriye avait insisté : «Dursune, amène ton mari aussi demain soir ». Au cours de la semaine suivante, Kadriye étant occupée par des invités, son mari Kazım s'est rendu chez Hüseyin et Dursune et nous avons pris un café tous ensemble dans la 
cuisine. Le lendemain nous sommes allés, Dursune, Hüseyin, Kazim dans un restaurant fast food: il s'agissait de présenter Hüseyin et moi, qui étions tous les deux des «nouveaux » dans le quartier (Dursune et Hüseyin avaient aménagé au cours de l'année et d'après son épouse, Hüseyin " [allait] trop souvent voir ses amis de l'ancien quartier, il ne cherche pas à se faire des amis ") à un autre habitant du quartier, Ahmet, décrit comme un homme sincère et qui "n'a pas sa langue dans la poche ». Un autre soir, les trois hommes se sont revus chez Kazım pour regarder un match de football télévisé. Tandis que les enfants jouaient dans les chambres, les femmes (les épouses des trois hommes et les deux jeunes filles de Ahmet) s'étaient retirées dans la cuisine après le premier verre de thé pris avec les hommes. Dursune était venue me dire à l'oreille : "Qu'est-ce que tu fais parmi les hommes? Viens parmi nous ». Une fois rentrés chez eux, dans la nuit, Dursune et Hüseyin discutent au salon :

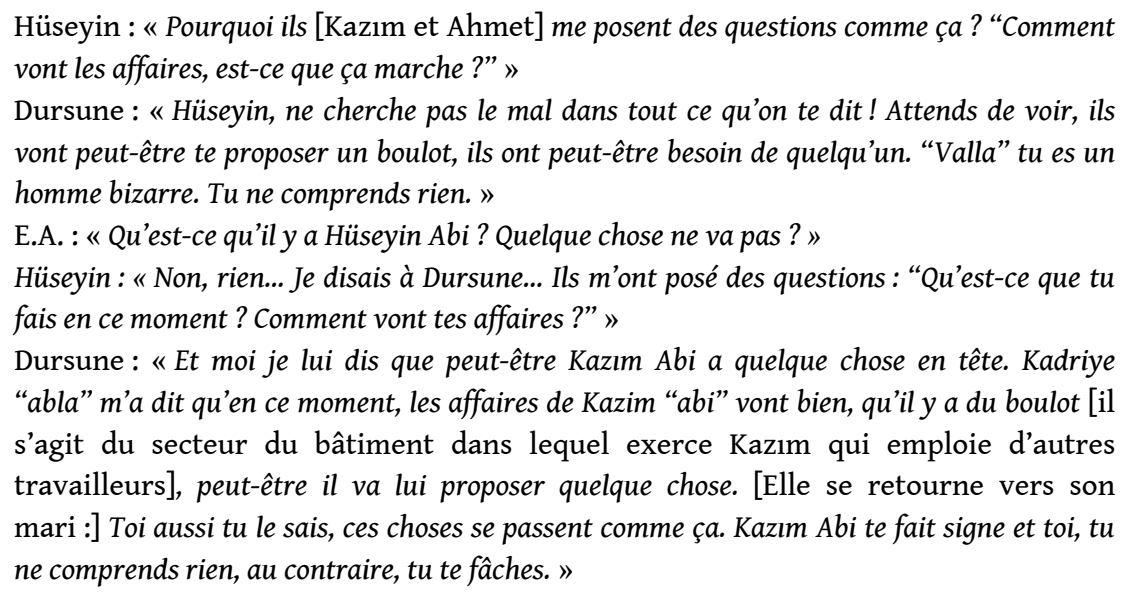

La proposition et la demande d'un emploi relèvent d'un processus où les rencontres se font d'une certaine manière suivant certains ordonnancements. Dans ce processus qui mène à l'accès à un emploi ou à de meilleures opportunités professionnelles intervient le réseau social des femmes.

\section{La distinction des femmes maghrébines}

Yasemin est la seule femme turque recevant chez elle une voisine "arabe", Sonia (fille issue d'un père algérien et d'une mère française, mariée à un homme d'origine marocaine), bien qu'elle ne soit jamais allée chez Sonia. Aux débuts de mes visites chez Yasemin, celle-ci s'efforçait de me faire comprendre, parfois en présence de Sonia qui ne comprenait pas le turc, le mépris qu'elle éprouvait à l'égard de celle-ci : «Elle [Sonia] est encore là, celle-ci ! », " Elle prend trop l'habitude de venir chez nous ». Elle me faisait aussi part du sentiment d'apitoiement qu'elle éprouvait à l'égard de Sonia, par lequel elle visait sans doute à rendre légitime cette situation hors norme :

«La famille de son mari l'exclut. Comme chez nous, on exclut les autres [épouses non turques des hommes turcs]. Elle n'a que nous... Il paraît que sa mère est une femme riche. Elle se fait faire les ongles quand elle va chez elle... Celle-là [Sonia] n'est pas dangereuse. Regarde-la, "karnı burnunda" ["son ventre va toucher son nez" : Sonia est enceinte de sept mois]. Bon, parfois elle nous montre son ventre [en soulevant son vêtement] devant Selçuk [le fils de Yasemin, âgé de 17 ans]. C'est parce qu'elle ne sait pas que chez nous c'est "ayıp". Mais je lui apprends les choses. Elle est devenue turque avec nous. Ici, les autres [femmes turques], elles ne prennent pas les femmes "Arabes" chez elles. À part une Turque, personne ne peut entrer chez elles. Elles pensent qu'elles vont "ayartmak" leurs 
maris [les provoquer à entretenir des relations extra conjugales]. Comme si leurs

maris ne faisaient pas ce qu'ils ont à faire ailleurs ». et «découvertes»(comme le qualificatif «sales», ces termes comportent une connotation négative) ${ }^{33}$ sont perçues par ces dernières comme une menace pour leurs relations conjugales et les futures alliances conjugales de leurs enfants. La maison est pour les femmes turques, un lieu sacré et contrôlé où se négocient l'avenir, le travail et le mariage. Si ces pratiques de distanciation et de distinction visent les Maghrébines, c'est parce qu'elles constituent une catégorie perçue comme étant relativement plus proche que les «Français », les "Gâvur » et les « cas sociaux ».

\section{En guise de conclusion} manière de trouver du travail, les activités des femmes entre elles sont des éléments importants et liés les uns aux autres. Ce système exclut notamment les alliances matrimoniales avec d'autres catégories. «Dominées » si l'on veut en ce qui concerne le «choix» de leur conjoint, les femmes jouent comme on l'a montré un rôle extrêmement actif mais peu visible de l'extérieur dans la régulation des rapports sociaux internes à la communauté turque et à l'intérieur du quartier. Conjuguant différents statuts, voisines, mère et fille, belle-mère et bru, sœurs, belles-sœurs, femmes issues du même village (köylüm), épouses de collègues, les femmes immigrées turques créent et entretiennent par leurs visites quotidiennes un réseau d'interconnaissance, de recueil d'informations, d'évaluation et de contrôle social entre femmes. La réputation qui s'établit ainsi (et se transforme) pour chaque femme a des conséquences sur le recrutement de la main-d'œuvre et sur le choix du conjoint de leurs enfants. Ce système écarte les femmes maghrébines du quartier et fonctionne en lien avec les villages d'émigration.

61 Ayant été ultérieurement aux villages où le même système de visite n'existe pas (visite quasi nulle entre femmes non-membres de la parentèle), on peut aussi conclure que les femmes immigrées turques ont inventé une forme d'adaptation à la situation nouvelle (l'émigration ayant rendu les rapports plus distants, les risques étant devenus moindres) qui permet un contrôle social de la communauté, un contrôle qui est moins directement celui des hommes que des femmes mariées sur les jeunes et les autres turcs. La pression de ceux qui sont aux villages est forte, et les immigrés sont dépendants des villages. Ceux-ci payent la mobilité sociale par un éloignement physique et non par un éloignement moral. Il n'est pas sûr que ce système puisse durer longtemps en considération du nombre croissant des divorces. 


\section{BIBLIOGRAPHIE}

ABU-ZEID A. (1966) Honour and shame among the Bedouins of Egypt, in J. G. Peristiany Eds., Honour and Shame. The Values of Mediterranean Society, University of Chicago Press, pp. 243-261. BECKER H. S. (2000) Enquête de Terrain : Quelques ficelles du métier, Sociétés Contemporaines (Travail de terrain et observations des comportements), 40, pp. 151-164.

BOURDIEU Pierre (1958) Sociologie de l'Algérie, Paris, PUF (coll. Que sais-je ?)

BOURDIEU Pierre (1966) The Sentiment of honour in Kabyle society, in J. G. Peristiany Eds., Honour and Shame. The Values of Mediterranean Society, University of Chicago Press, pp. 191-241. BOURDIEU Pierre (1972) Esquisse d'une théorie de la pratique, Paris, Seuil. GEERTZ Cliford (1999 [New York, 1983) Savoir local, savoir global. Les lieux du savoir, Paris, PUF. GÖLE Nilüfer (1993) Musulmanes et Modernes. Voile et civilisation en Turquie, Paris, la Découverte. HOROWITZ R. (1983) Honor and the American Dream. Culture and Identity in a Chicano Community, Rutgers University Press, New Brunswick, NJ.

HOROWITZ R. (1986) Remaining an outsider. Membership as a threat to research rapport, Urban life, 14 (4), pp. 409-430.

HUGHES E. C. (1971) The Sociological Eye. Selected Papers, Chicago, Aldine.

KASTORYANO Riva (1986) Ettre Turc en France. Réflexions sur familles et communauté, Paris, CIEMI et L'Harmattan.

LEPOUTRE David (1997) Cour de banlieue. Codes, rites et langages, Paris, Odile Jacob.

LOPATA H. Z. (1971) Occupation : Housewife, Oxford University Press.

LOPATA H. Z. (1976) Polish Americans. Status Competition in an Ethnic Community, Prentice-Hall, Englewood Cliffs, New Jersey.

MAUSS Marcel (2001 [1950]) Sociologie et anthropologie, Paris, PUF (coll. Quadrige).

PERISTIANY J. G. (1966) Honour and shame in a Cypriot highland village, in J. G. Peristiany Eds., Honour and Shame. The Values of Mediterranean Society, University of Chicago Press, pp. 171-190.

PITT-RIVERS Julian (1966) Honour and social status, in J. G. Peristiany Eds., Honour and Shame. The Values of Mediterranean Society, University of Chicago Press, pp. 19-77.

PITT-RIVERS Julian (1997 [Cambridge Univ. Press, 1977]) Anthropologie de l'honneur. La mésaventure de Sichem, Paris, Hachette.

RIGONI Isabelle (2000) Introduction, in I. Rigoni Coord., Turquie : les mille visages. Politiques, religion, femmes, immigration, Paris, Syllepse, pp. 7-21.

ROGERS S. C. (1975) Female forms of power and the myth of male dominance : a model of female/ male interaction in peasant society, American Ethnology, 2 (4), pp. 728-755.

SAYAD Abdelmalek (1999) La Double absence. Des illusions de l'émigré aux souffrances de l'immigré, Paris, Seuil.

TAPIA Stéphane de (1995) La France dans l'espace transnational turc, Colloque national de démogéographie de Poitiers, 25-26-27/10/1995. 
THOMAS W. I. \& ZNANIECKI F. (1998 [Chicago, 1919]) Le Paysan polonais en Europe et en Amérique. Récit de vie d'un migrant, Paris, Nathan (coll. Essais \& Recherches).

TIMMERMANS Christiane (1994) Jeunes filles de Turquie. Vie familiale et instruction scolaire, in N. Bensalah Dir., Familles turques et maghrébines aujourd'hui, Paris, Academia \& Maisonneuve et Larose.

TRIBALAT Michèle (1995) Faire France, Paris, La Découverte (coll. Essais).

WHITE W. F. (1943) Street Corner Society, Chicago, University of Chicago Press.

\section{NOTES}

1. Nom modifié afin de préserver l'anonymat.

2. Selon Kastoryano (1986: 63 et 44), ces visites "entraînant un retour au traditionalisme" constituent « un secours à l'isolement et à la solitude» des femmes "renvoyées à la seule existence du groupe ». Timmermans (1994: 178) affirme que les femmes immigrées turques trouveraient ainsi "de l'amitié et de l'affection principalement auprès des personnes de leurs propres sexes ». Elle remarque que «seules les tâches ménagères que leurs filles auront à effectuer dans leur ménage sont considérées comme utiles et bonnes à apprendre». Les jeunes filles qui "passent le plus clair de leur temps à regarder la télévision ou rendent visite à d'autres femmes » seraient (comme leurs mères) « soumises au système des valeurs de la société traditionnelle turque » (Timmermans, $1994: 180 ; 181 ; 186)$.

3. Il s'agit d'un nom fictif attribué à la commune à laquelle est rattaché administrativement le quartier des Lilas.

4. Lorsque les habitants musulmans du quartier emploient le qualificatif Gâvur (en turc: mécréant, qui ne professe pas la vraie foi, non-musulman, Chrétien, Européen, Occidental) ou Gawour(ia) (en arabe), c'est un(e) « Français(e)» qui est évoqué(e).

5. Le fait qu'il m'ait été possible de faire un tel micro-recensement est évidemment un indice de la forte intégration (ou du moins un haut degré d'interconnaissance) de la population d'origine turque.

6. Au cours de l'étude, «turc » désigne toute personne de nationalité turque et/ou française qui se dit «turque».

7. Les âges des trois enfants d'une famille ayant aménagé au cours de ma recherche dans le quartier ne sont pas connus. Les habitants ne disposaient pas d'information sur « les nouveaux venus ", si ce n'est qu'ils avaient deux fils et une fille.

8. Il s'agit de la première étape - la seconde étant les fiançailles et la dernière, le mariage - du processus par lequel la relation entre deux célibataires acquière une reconnaissance sociale.

9. La langue turque permet ici un jeu de mot. Le fait même de saluer les femmes turques par cette formule les conduit à parler des activités ménagères.

10. «Aux cafés turcs, c'est comme la bourse. Ils [les hommes turcs] négocient entre eux le travail et l'échange de main-d'oeuvre», affirme un témoignage.

11. Misafir signifie «l'invité » (plus précisément une personne reçue, qu'elle soit invitée ou non). Alors que Misafirperver (« qui aime le misafir ») signifie hospitalier et Misafirperverlik, l'hospitalité, Misafirlik est l'institution qui permet à deux individus habitant dans des logements distincts de concevoir leur relation comme un échange d'hospitalité en se rendant l'un chez l'autre (l'un reçoit l'hospitalité en rendant visite/l'autre donne l'hospitalité en recevant la visite). Misafirlik correspond à une situation relationnelle et peut mettre en jeu la présence de plus de deux individus quand plusieurs hôtes invités sont réunis chez un hôte invitant. Ce sont les femmes qui vont les unes chez les autres pour le misafirlik, accompagnées ou non de leurs maris et leurs enfants. 
12. Dans Occupation: Housewife, Lopata (1971) accorde un intérêt particulier aux rapports de voisinage entre femmes envisagés comme production du lien social.

13. Les prénoms sont suivis de termes d'adresse ou qualificatifs comme Abla (grande sœur) et Abi (grand frère), y compris dans un discours formulé en français et même en cas d'absence de liens de parenté ; les cadets expriment ainsi le sentiment de respect aux aînés quand ils s'adressent à eux.

14. Ces invitations formelles peuvent selon les affinités devenir moins formelles, comme le remarque Lopata dans les classes supérieures (1971:249): «Formal rituals have been developed, mostly among the upper classes, which guarantee at least minimal neighboring. These usually begin with the traditional "calling on" new neighbors, within the prescribed times and with normatively controlled behavior. Etiquette demands a return call, which is often followed by invitations to pre-arranged "events" in homes or other socializing locations. [...] The invitations are returned and the relation is built up by an increase in the frequency and variety of occasions in which all are involved. [...] The deepening of selectively chosen friendships among neighbors may involve expansion of the form and an increase in informality ».

15. Il existe dans la langue turque une formule dont l'usage est exclusivement réservé à cette situation : iadeyi ziyaret (rendre une visite pour rendre la visite rendue). Cette formule permet aussi de présenter la visite à ceux qui la reçoivent comme un échange de bon procédé, un acte de politesse (comme l'observe Mauss chez les clans Polynésiens (2001:258) : "L'invitation doit être rendue, tout comme la "politesse"»), une visite rendue pour "ne pas déprécier» l'autre et n'engageant pas la réputation de celui qui la rend. «Les paysans espagnols utilisent communément la formule "para no depreciarlo" lorsqu'ils acceptent la nourriture ou la boisson qu'on leur offre. De cette manière l'invité se décharge du soupçon de goinfrerie et d'indélicatesse en prétendant ne rien accepter que par respect pour son hôte. » (Pitt-Rivers, 1997 :169).

16. Humiliant ou déshonorant : «To be dishonoured is to be rejected from the role to which one aspired. 'I am who I am' is answered: 'You are not who you think you are' » (Pitt-Rivers, 1966: 72).

17. Le «souci de réputation » exprimé par le « qu'en dira-t-on» ou le « que dirán " (Pitt-Rivers, 1997 : 49) est présent aussi bien dans le système de valeurs du pueblo andalou, des paysans grecs (Peristiany, 1966), des Bédouins d'Egypte (Abu-Zeid, 1966), des Kabyles (Bourdieu, 1966 ; 1972)... que dans Street Corner Society étudié par White (1943), le Chicano Community par Horowitz (1983) ou les clans polynésiens. [Peristiany $(1966: 66)$ suggère que les premiers anthropologues auraient en effet pu traduire le mot mana par honneur et indique que Mauss avait en effet suggéré cette traduction]. Honneur/honte, horma, vergüenza, timē, saraf, ces sentiments ont été perçus souvent du seul point de vu des hommes et "l'honneur» des femmes dans son rapport à l'honneur des hommes - qu'elles soient objets de transactions et de luttes d'honneur entre hommes (le transfert de la responsabilité de l'honneur d'une femme, de son père et ses frères à son mari ; déshonorer son ennemi en s'acquittant de sa femme ou honorer son hôte en lui offrant sa femme) ou qu'elles tirent du contrôle de leur pureté sexuelle un pouvoir sur les hommes dont les honneurs sexuels en dépendent. Le point de vue «androcentrique » souvent adopté par les études menées sur les communautés villageoises en général repose aussi, comme le remarque Rogers (1975), sur une vision modernisante présumant la subordination des femmes aux hommes dans les sociétés traditionnelles paysannes. Quelques travaux ont toutefois suggéré que dans ce type de sociétés la domination masculine n'est qu'une façade derrière laquelle se cache le pouvoir des femmes sur les hommes. Rogers (1975), se demandant pourquoi le pouvoir des femmes paysannes est un pouvoir dissimulé, suggère, à l'appui de ses propres recherches menées dans un village en France, que la domination masculine constitue en effet un mythe dont la perpétuation convient à la fois aux hommes et aux femmes, permettant ainsi un équilibre des rapports de pouvoirs entre les deux groupes, qui sont interdépendants.

18. À l'exemple des immigrés polonais aux États-Unis, très engagés dans la compétition de statut, la possession de voitures, de maisons, etc. ne constitue pas le mode exclusif de l'accumulation des 
" points de statut »: "[...] the behavior of people who are identified with them is used by others as a criterion of their own reputation " (Lopata, $1976: 9$ ).

19. " "I am who I am" subsumes "whom I am associates with" " (Pitt-Rivers, 1966 : 35).

20. En langue turque, «étranger» (Yabancl) signifie également «celui qui n'a pas encore acquis l'habitude, les connaissances, l'expérience dans un domaine ».

21. Outre le terme de « citadine » que les adultes employaient à mon sujet, les enfants utilisaient ce qualificatif pour exprimer ma situation d'intruse dans le milieu. Le terme se réfère à l'image généreusement exploitée par le cinéma turc des années 1980 de la femme appar-tenant à «la haute société ».

22. Pour le processus de la formation et de la transformation de ces identités ainsi que l'interaction de celui-ci avec l'évolution de la recherche, voir Horowitz (1986).

23. Namus est défini en turc comme : "L'attachement fort aux règles d'honneur et morales au sein de la société, loyauté, vertu»; traduit en français : "Honneur, honorabilité, honnêteté, pudeur, chasteté (femmes)». Ma position de mi-hôte/mi-membre de famille m'engageait à protéger l'honneur que je partageais avec les autres membres (il existe en turc un éventail de termes pour signifier l'honneur - onur, gurur, şeref, şan, namus - qui ne sera pas examiné ici).

24. L'honneur commun peut concerner toutes les catégories auxquelles l'individu s'identifie et à travers lesquelles il opère la distinction entre «nous » et « eux » : la famille, la communauté, la nation (Peristiany, 1966 : 173). En m'acceptant chez elle, cette femme relevait un défi qui était perçu par tous les Turcs du quartier.

25. «Ainsi, lorsque quelqu'un avançait que les Nègres sentent plus mauvais que les Blancs, ces bonnes âmes malavisées s'efforçaient de prouver que les Blancs sont, en réalité, incapables de sentir la différence entre la sueur d'un Blanc et la sueur d'un Noir. Et ces chercheurs furent positivement ravis de voir que leurs travaux montraient également que les Américains d'origine chinoise trouvaient que la sueur des Blancs sentait particulièrement mauvais. Pour Hughes, ces chercheurs se sont égarés parce qu'ils n'apercevaient pas la logique d'argumentation qu'ils cherchaient à combattre. [...]» (Becker, $2000: 152)$.

26. Villages des départements d'Erzurum, Bayburt, Samsun, Sivas situés dans les régions Nord et Nord-Est de la Turquie.

27. Les statistiques sur les catégories de couples concernent 31 couples en excluant un ménage de la liste des familles turques (installé depuis peu de temps dans le quartier). Cependant l'échantillon comporte 32 couples car le couple où l'époux est en Turquie (le cas de la jeune fille de 15 ans mariée habitant chez ses parents) a été intégré dans l'analyse statistique des couples (ce couple figure dans la catégorie C2). Le couple turco-maghrébin ne figurant pas dans la liste des familles turques n'est pas considéré dans les catégories de couple.

28. Görmek signifie « voir » et Gören : « celui qui voit ». Le terme de Görücü s'applique à la sphère matrimoniale parce qu'il désigne ceux qui, étant présents chez la famille de la jeune célibataire, «voient » celle-ci afin de l'« évaluer » et la demander en mariage pour leur fils. La définition du dictionnaire est la suivante : «Görücü, adj. 1.Voyant, qui voit. 2. Femme envoyée de la part d'un homme qui veut se marier, pour chercher ou voir une jeune fille», Pars Tuğlac1, Türkçe-Fransızca Sözlük [Dictionnaire Turc-Français], Istanbul, İnkilâp.

29. Comme le nisba au Maroc, qui «classe, mais ne définit pas [et] laisse le reste, c'est-à-dire presque tout, qui devra être complété par le processus de l'interaction elle-même » (Geertz 1999 : 87).

30. "[...] there must be a community within which his "reputation" is known, that is, a community of people who share status hierarchies and know him or her well enough to undertake the process of ranking and of communicating the ranks to each other. This is one of the major functions of gossip, the better known one being social control. [...] If we stick the criterion of "reputation", we see that there is no need to limit status competition to territorially bound communities » Lopata (1976:10).

31. Les données que nous avons collectées ne nous ont cependant pas permis d'examiner la manière dont ces réputations se négocient dans l'ensemble de l'okolica.-“[...] what Thomas and 
Znaniecki (1918-1920) called the okolica [...] is the area within which a person's reputation is contained, the social area in which the person lives and interacts, the social life space which contains his identities. The okolica of the peasant includes the familiy, home, lands, the village of families, and somethimes other villages" Lopata (1976: 19).

32. Ayıp diffère de vergüenza : ce n'est pas un sentiment mais un comportement, verbal ou physique (il y a aussi des films dits ayıp et des parties du corps dits ayıp) ; qui est perçu par les autres comme une faute suscitant critique mais n'affligeant pas un déshonneur à son auteur : "If a woman should wear short clothes, the action is called 'aib'. [...] Actions of 'aib' do not usually require severe intervention in the part of society as a whole and any response is usually directed against the wrongdoer himself. The wrongdoer may be blamed or mocked and ridiculed or simply taken as a joke" (Abu-Zeid,1966: 246).

33. Produits d'un contexte donné, les ingrédients d'un étiquetage peuvent aussi bien trouver leur sens en situation d'enquêté/enquêteur (de sexe féminin, perçu comme "cultivée ", " citadine ", «moderne » etc.). Au milieu des années 1980, une femme turque résidant dans un quartier HLM affirmait à Kastoryano au sujet de ses voisines maghrébines : "Nous, on ne s'entend pas avec elles, elles portent déjà le fichu, et leurs enfants sont délinquants » (Kastoryano, 1986:52).

\section{AUTEUR}

\section{ELIF AKSAZ}

Doctorante à Paris 1 et membre du GETI à Paris 8. Université Paris 1, Centre Pierre Mendès-

France, Bureau B 14-06, 90, rue de Tolbiac, 75013 PARIS. 